\title{
Experimental Results and Evaluation of SmartBox Stimulation Device in a P2P E-learning System
}

\author{
Keita Matsuo $\nmid$, Leonard Barolli $\nmid$, Joan Arnedo-Moreno $\nmid \dagger$, Fatos Xhafa $\nmid \ddagger$, \\ Akio Koyama ${ }^{\ddagger \dagger}$, Arjan Durresi ${ }^{\ddagger \ddagger}$ \\ †Graduate School of Engineering, Fukuoka Institute of Technology (FIT) \\ 3-30-1 Wajiro-Higashi, Higashi-Ku, 811-0295 Fukuoka, Japan \\ E-mail: bd07002@bene.fit.ac.jp \\ $\ddagger$ Department of Information and Communication Engineering \\ Fukuoka Institute of Technology (FIT) \\ 3-30-1 Wajiro-Higashi, Higashi-Ku, 811-0295 Fukuoka, Japan \\ E-mail: barolli@fit.ac.jp \\ $\dagger \dagger$ Department of Computer Science, Multimedia and Telecommunication \\ Universitat Oberta de Catalunya \\ Rambla del Poblenou, 15608018 Barcelona, Spain \\ E-mail: jarnedo@uoc.edu \\ $\dagger ‡$ Department of Languages and Informatics Systems \\ Technical University of Catalonia \\ Jordi Girona 1-3, 08034 Barcelona, Spain \\ E-mail: fatos@1si.upc.edu \\ $¥ \dagger$ Department of Informatics, Yamagata University \\ 4-3-16 Jonan, Yonezawa 992-8510, Yamagata, Japan \\ E-mail: akoyama@eie.yz.yamagata-u.ac.jp \\ ${ }_{\ddagger}$ Department of Computer and Information Science \\ Indiana University Purdue University at Indianapolis (IUPUI) \\ 723 W. Michigan Street SL 280, Indianapolis, IN 46202, USA \\ E-mail: durresi@cs.iupui.edu
}

\begin{abstract}
In this paper, we present the experimental results and evaluation of the SmartBox stimulation device in $P 2 P e$ learning system which is based on JXTA-Overlay. We also show the design and implementation of the SmartBox environment that is used for stimulating the learners motivation to increase the learning efficiency. The SmartBox is integrated with our $P 2 P$ system as a useful tool for monitoring and controlling learners' activity. We found by experimental results that the SmartBox is an effective way to increase the learner's concentration. We also investigated the relation between learner's body movement, concentration, and amount of study. From the experimental results, we conclude that the use of SmartBox is an effective way to stimulate the learners in order to continue studying while maintaining the concentration.
\end{abstract}

\section{Introduction}

Recently, many new devices and networks are appearing and in the past few years we have observed an explosive growth of multimedia computing, communication and applications.

The Internet is growing every day and the performance of computers is increased exponentially. However, the Internet architecture is based on Client/Server (C/S) topology, therefore can not use efficiently the clients features. It should be noted that computers of today have the same characteristics as old super-computers. Also, with appearance of new technologies such as ad-hoc networks, sensor networks, body networks, home networking, new network devices and applications will appear. Therefore, it is very important to monitor, control and optimize these network devices via communication channels. However, in large-scale networks such as Internet, it is very difficult to control the network devices, because of the security problems. 
In order to make the networks secure many security devices are used. The firewalls are used for checking the information between private and public networks. The information is transmitted according to some decided rules and it is very difficult to change the network security policy. There are many small networks and Intranets that do not allow the information coming from other networks. Therefore, recently many researchers are working on Peer-to-Peer (P2P) networks, which are able to overcome the firewalls, NATs and other security devices without changing the network policy. Thus, $\mathrm{P} 2 \mathrm{P}$ architectures will be very important for future e-learning systems.

Virtual campuses and virtual universities are new ways of organizing students activities by using IT technologies. The virtual university is one of the most widely used form of virtual organization in today's teaching and learning environment. However, most of the current virtual organizations use server-mediated Internet-based applications that enable sharing among the members of the community.

In $\mathrm{C} / \mathrm{S}$ approaches shared resources are centralized on servers and members of the virtual community (students, teachers, researchers, etc.) access them through Web clients. Thus, everything is done at server side while the client side just an interface. In general, server-mediated approaches show several drawbacks, such as lack of scalability and efficiency in virtual campuses of thousands of students.

The most powerful alternative to server-based approaches for applications in virtual universities is the use of decentralized approaches such as P2P systems. In such systems, the computational burden of the system can be distributed to peer nodes of the system. Therefore, in decentralized systems users become themselves actors by sharing, contributing and controlling the resources of the system. This characteristic makes $\mathrm{P} 2 \mathrm{P}$ systems very interesting for the development of decentralized applications $[1,2]$ for virtual campuses and virtual organizations.

During the last decade, many e-learning systems have been proposed and implemented, mostly using web technologies. With emerging of technologies such as P2P, sensor and ad-hoc networks, it will be possible to build ubiquitous systems that can offer an equal educational environment regardless the region, age, time or the place $[3,4]$.

In this paper, we present the experimental results and evaluation of the SmartBox stimulation device in P2P elearning system which is based on JXTA-Overlay. We also show the design and implementation of the SmartBox environment that is used for stimulating the learners motivation to increase the learning efficiency. The SmartBox is integrated with our P2P system as a useful tool for monitoring and controlling learners' activity.

The structure of this paper is as follows. In Section 2, we introduce the related work. In Section 3, we introduce the Project JXTA and JXTA-Overlay. In Section 4, we present the proposed $\mathrm{P} 2 \mathrm{P}$ e-learning system. In section 5, we discuss the experimental result. Finally, conclusions and future work are given in Section 6.

\section{Related Work}

In this section, we discuss the related work for P2P systems and Web based e-learning systems.

Much of the initial research efforts and projects on P2P systems were concerned with the investigation of overlay networks. It should be noted that each time more, this effort is oriented towards the design and implementation of libraries and platforms to support the development of real P2P applications, which could actually combine both Grid and P2P technologies. Bal et al. [5] motivated the need for research from conceptual, algorithmic and application level tools to facilitate the application development task, that is, tools that enable writing, deploying and running Grid/P2P applications.

Since in $\mathrm{P} 2 \mathrm{P}$ applications file sharing plays an important role, a lot of research effort is being devoted to the development of reliable file sharing systems to be used in P2P applications [6]. In particular, several studies are done for measuring the efficiency in $\mathrm{P} 2 \mathrm{P}$ systems $[7,8]$ as it is crucial.

Due to the opportunities provided by the Internet, more and more people are taking advantage of online distance learning courses. During the last few years enormous research efforts have been dedicated to the development of elearning systems. Consequently, many large projects have been established $[9,10,11,12,13,14,15]$. However, in these systems the e-learning completion rate is low. One of the reasons is the low study interest when the learner studies the subjects. Therefore, it is very important to stimulate learner's motivation during the study. In our previous works $[16,17]$, we proposed an agent based distance learning system to deliver appropriate studying materials to learners and stimulate learner's motivation.

However, all these systems are based on C/S architecture. In such approach the shared resources are centralized on servers and members of the virtual community (clients) access them through requesting protocols. Among the most important limitations of server-mediated approaches, we could distinguish lack of scalability, lack of fault tolerance, usually low performance/bottlenecks as well as high costs in acquiring, developing and maintaining such applications.

\section{JXTA Technology and JXTA-Overlay}

\subsection{JXTA Technology}

JXTA [4] technology is a generalized group of protocols that allow different devices to communicate and collaborate among them. JXTA offers a platform covering basic needs in developing P2P networks [18].

By using the JXTA framework, it is possible that a peer in a private network can be connected to a peer in the Internet by overcoming existing firewalls as shown in Fig. 1. In this figure, the most important entity is the router peer. A router peer is any peer which supports the peer endpoint protocol, 


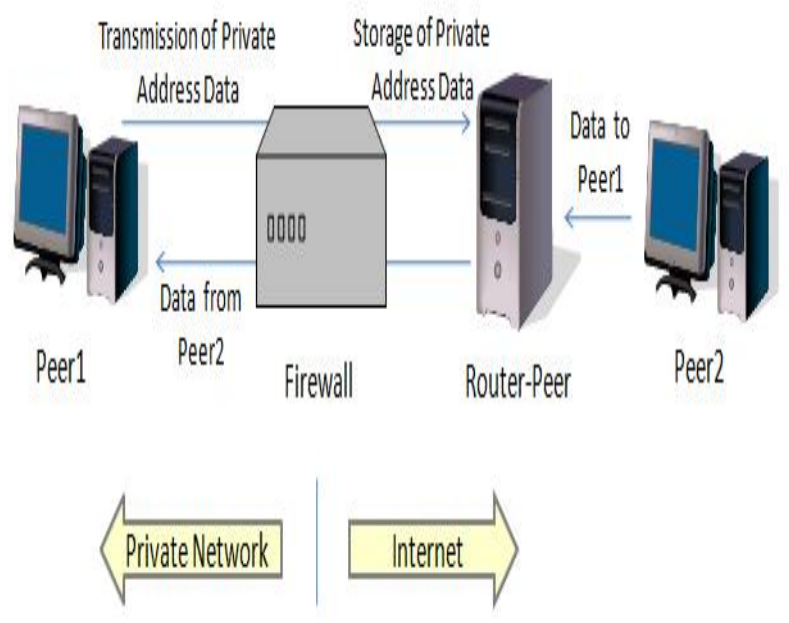

Figure 1. P2P communication.

routing messages between peer in the JXTA networks. The procedure to overcome the firewall is as follows.

- In the Router Peer is stored the private address of Peer1 by using the HTTP protocol to pass the firewall from Peer1.

- The Router Peer receives the data from Peer2 and access the Private address of Peer1 to transmit the data.

JXTA is an interesting alternative for developing P2P systems and groupware tools to support online teams of students in virtual campuses. In particular, it is appropriate for file sharing given that the protocols allow to develop either pure or mixed P2P networks. This last property is certainly important since pure $\mathrm{P} 2 \mathrm{P}$ systems need not the presence of a server for managing the network.

\subsection{JXTA-Overlay}

JXTA-Overlay project is an effort to use JXTA technology for building an overlay on top of JXTA offering a set of basic primitives (functionalities) that are most commonly needed in JXTA-based applications. The proposed overlay comprises the following primitives:

- peer discovery,

- peer's resources discovery,

- resource allocation,

- task submission and execution,

- file/data sharing, discovery and transmission,

- instant communication,

- peer group functionalities (groups, rooms etc.),

- monitoring of peers, groups and tasks.

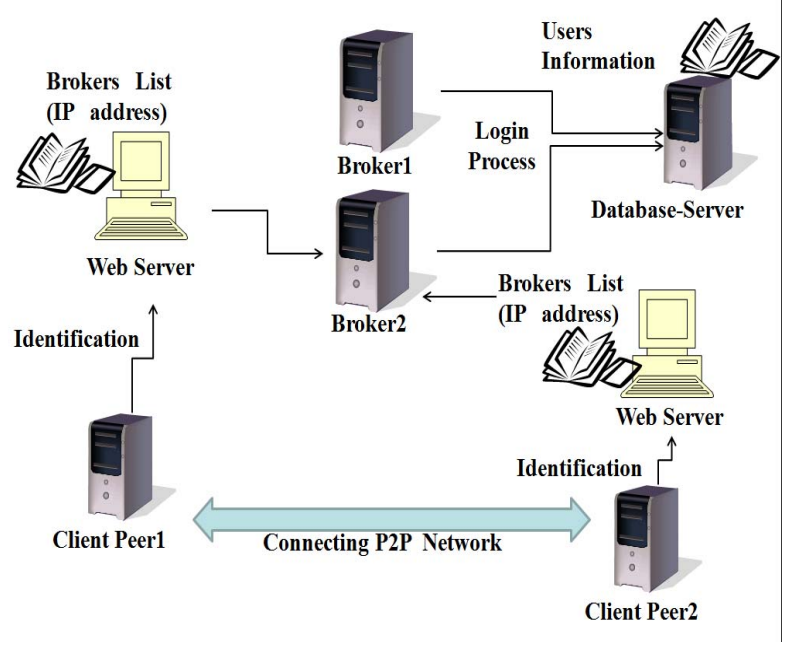

Figure 2. Structure of JXTA-Overlay system.

This set of basic functionalities is intended to be as complete as possible to satisfy the needs of JXTA-based applications. The overlay is built on top of JXTA layer and provides a set of primitives that can be used by other applications, which on their hand, will be built on top of the overlay, with complete independence. The JXTA-Overlay project has been developed using the ver-2.3 JXTA libraries. In fact, the project offers several improvements of the original JXTA protocols/services in order to increase the reliability of JXTA-based distributed applications [1] and to support group management and file sharing.

The architecture of the $\mathrm{P} 2 \mathrm{P}$ distributed platform we have developed using JXTA technology has two main peers: Broker and Client. Altogether these two peers form a new overlay on top of JXTA. The structure of JXTA-Overlay system is shown in Fig. 2.

\subsection{Internal Architecture of JXTA-Overlay}

Except Broker and Client peers, the JXTA-Overlay has also SimpleClient peers as shown in Fig. 3. The control layer interacts with the JXTA layer, and is divided into two parts: a lower part with functionality common to any kind of peer, and a higher part with functionality specific to Brokers and Clients.

- The common part provides functionality for doing JXTA messaging, discovery and advertisement.

- The Broker specific part provides functionality for managing groups of Brokers and keeping broker statistics.

- The Client specific part provides functionality for managing groups of Clients, keeping client statistics, managing its shareable files, managing the user configuration and creating the connection with a Broker. 


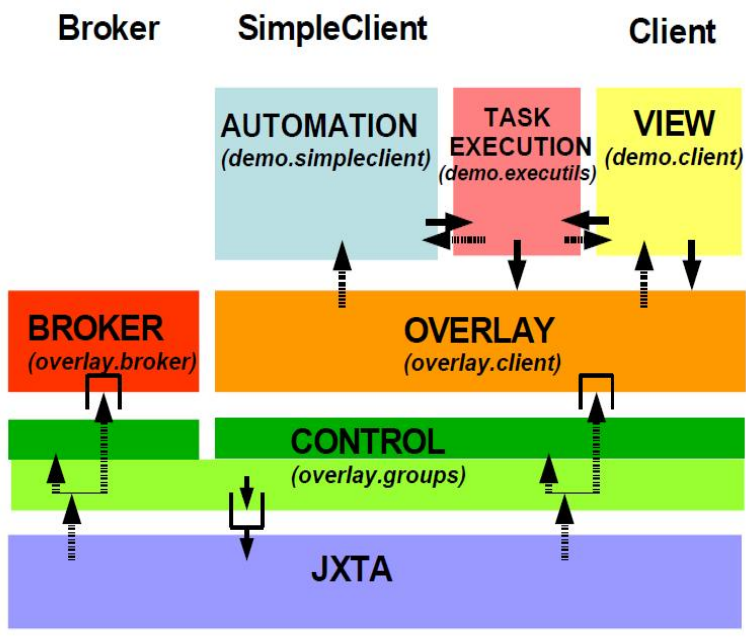

Figure 3. Internal Architecture of JXTAOverlay.

The lower part enqueues the JXTA messages to be sent. Whenever a message arrives, the JXTA layer fires an event to the lower layer, which in turn fires a notifications to the upper layers.

\section{Proposed P2P e-Learning System}

\subsection{Transmission Control and Management in JXTA-Overlay}

The most important part in e-learning systems or in virtual campus is the communication between peers (groups of students) and between teachers and students. By using the proposed JXTA-Overlay, it is possible to overcome, firewalls, routers, NATs, and bridges in the private networks. We explain in following the message transmission by the JXTA-Overlay.

JXTA-Overlay uses Universally Unique Identifier (UUID) in order to identify the peers in the private network from the Internet. The UUID is a general unique identifier. It is generated by the NIC address of the computer, date and time. By knowing the UUID and TCP address, it is possible to make address translation.

We implemented a control system that is able to control a peer in a private network from a peer in the Internet as showing in Fig. 4. The control targets are considered the network devices such as RS232C port, LPT port and USB port. By implementing this kind of control system, we are able to collect data and control the peers in a Wide Area Network (WAN). Thus, we will be able to control all devices that are connected to the peers. We control the USB devices and RS232C equipment. This is because USB devices are very popular and are used almost in every computer. Also, by using USB it is possible to control motors and LEDs. The

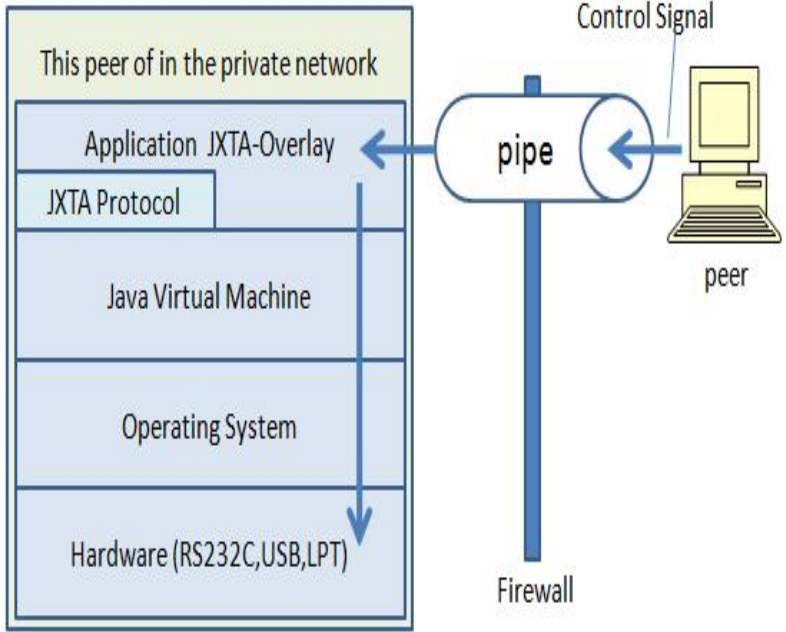

Figure 4. Implementation of remote control in JXTA-Overlay.

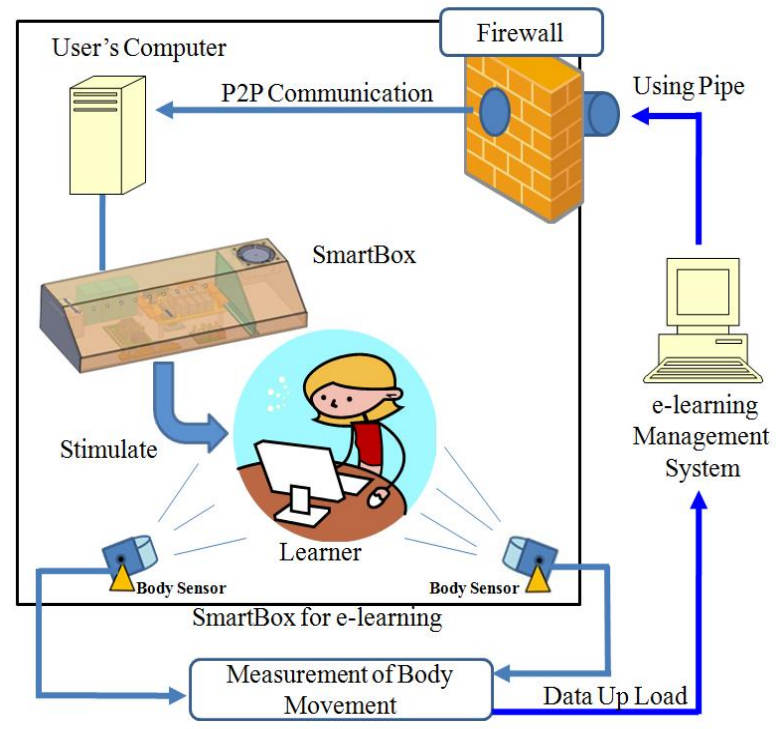

Figure 5. Implemented P2P e-learning system.

RS232C is a legal interface and many devices have implemented it.

\subsection{Implementation of P2P e-Learning System}

In our proposed system we combine P2P, Web and Sensor technologies. We implemented a P2P e-learning system as shown in Fig. 5. Our goal is not only to monitor and control the students' activity in a WAN but also to stimulate and increase learners' motivation. For this reason, we proposed and implemented a SmartBox as shown in Fig. 6. The SmartBox is integrated with our P2P system as a useful tool for monitoring and controlling learners' activity. 


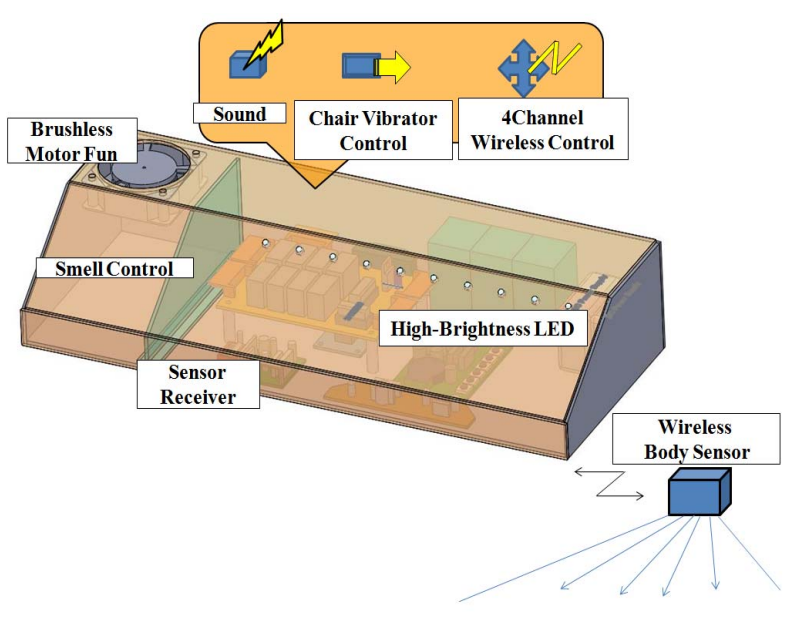

Figure 6. SmartBox functions.

Previously, we implemented a SmartBox device that was big in size and difficult to be moved in different places. The size of previous SmartBox was $50 \times 10 \times 15 \mathrm{~cm}$. In order to occupy a small space in the learner's desk and to transport easily in different places we designed and implemented a new SmartBox. The size of the new SmartBox is $35 \times 7 \times 12$ $\mathrm{cm}$. The SmartBox is equipped with different sensors (for sensing learner situation) and devices (used for stimulating learner's motivation).

The SmartBox has the following sensors and functions.

- Body Sensor: for detecting the learner's body movement.

- Chair Vibrator Control: for vibrating the learner's chair.

- Light Control: for adjusting the room light for study.

- Smell Control: for controlling the room smell.

- Sound Control: to emit relaxing sounds.

- Remote Control Socket: for controlling AC 100V socket (on-off control).

A snapshot of the previous SmartBox and new SmartBox is shown in Fig. 7. The use of SmartBox for e-learning is shown in Fig. 8. The SmarBox can detect the learners body movement by using body sensor.

\section{Experimental Results}

For the investigation of effects of the SmartBox, we used an easy arithmetic operation as learning material. as shown in Fig. 9. We carried out the experiments for two cases: when using SmartBox and not using SmartBox. We deal with the following issues.

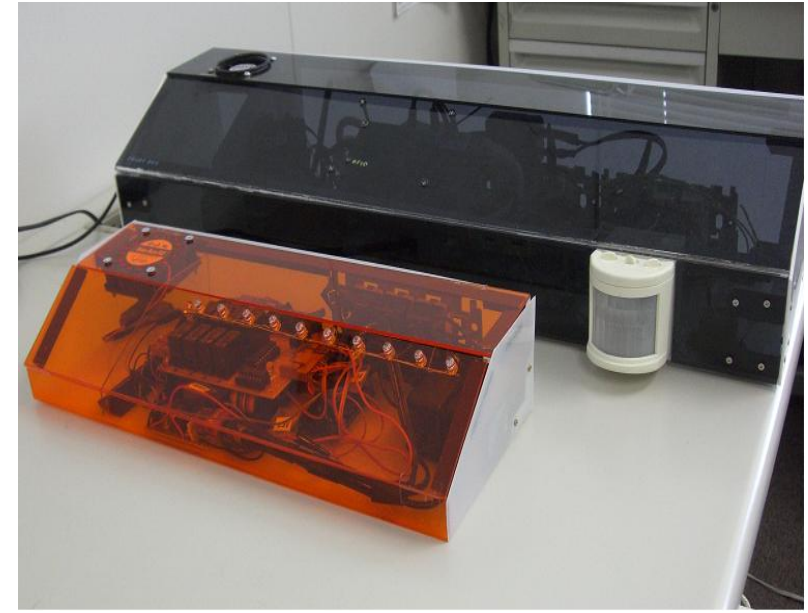

Figure 7. A snapshot of new SmartBox (Front) and previous SmartBox (Back).

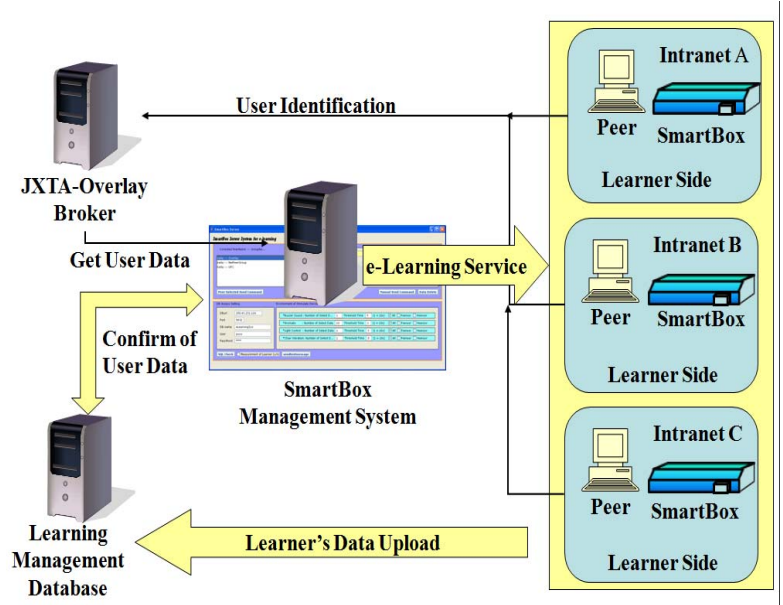

Figure 8. System structure when SmartBox is used for e-learning.

- Measurement of learners' body movement when using SmartBox and not using SmartBox.

- Comparison of stimulation effects when using light and sound.

- Investigation of relation between learner's body movement and amount of study.

\subsection{Body and Hand Movement}

The proposed system can detect the learner's movement by using body sensors. The measurement data for learners' body movements are shown in Table 1 and Fig. 10. We got these data after observing 41 learners. After a sensor 


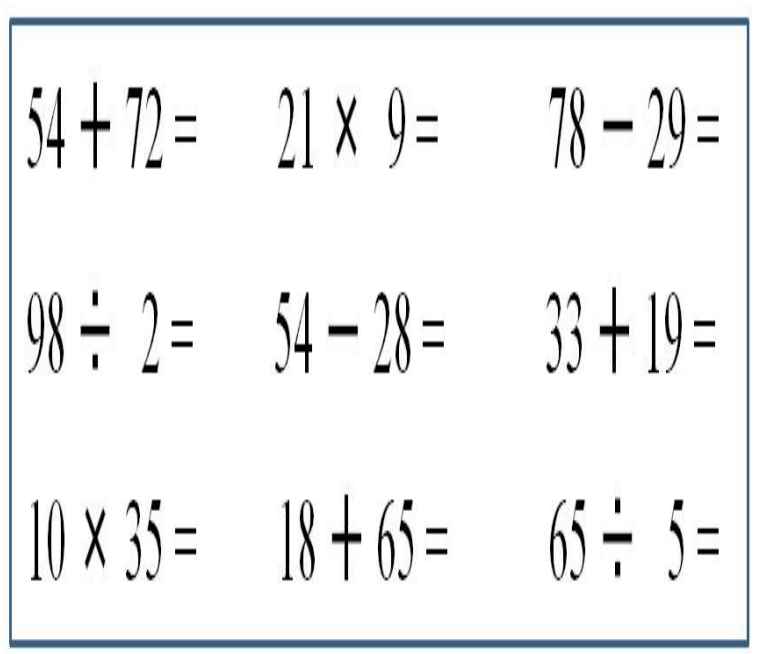

Figure 9. Learners' material for experiments.

Table 1. Reactions rate of body sensor when using SmartBox and not using SmartBox.

\begin{tabular}{|c|c|c|}
\hline $\begin{array}{c}\text { Response Range } \\
\text { Value }(\mathrm{sec})\end{array}$ & $\begin{array}{c}\text { Using SmartBox } \\
\text { Reaction Rate }\end{array}$ & $\begin{array}{c}\text { Not Using SmartBox } \\
\text { Reaction Rate }\end{array}$ \\
\hline 0s-10s & 67.32 & 56.12 \\
\hline $10 \mathrm{~s}-20 \mathrm{~s}$ & 22.70 & 25.25 \\
\hline $20 \mathrm{~s}-30 \mathrm{~s}$ & 6.14 & 8.46 \\
\hline $30 \mathrm{~s}-40 \mathrm{~s}$ & 2.34 & 3.52 \\
\hline $40 \mathrm{~s}-50 \mathrm{~s}$ & 0.82 & 1.78 \\
\hline $50 \mathrm{~s}-60 \mathrm{~s}$ & 0.18 & 1.07 \\
\hline $60 \mathrm{~s}-70 \mathrm{~s}$ & 0.06 & 0.81 \\
\hline $70 \mathrm{~s}-80 \mathrm{~s}$ & 0.05 & 0.59 \\
\hline $80 \mathrm{~s}-90 \mathrm{~s}$ & 0.03 & 0.38 \\
\hline $90 \mathrm{~s}-100 \mathrm{~s}$ & 0.02 & 0.34 \\
\hline $100 \mathrm{~s}-110 \mathrm{~s}$ & 0.02 & 0.29 \\
\hline
\end{tabular}

reacted, the interval time was reset to zero. We consider where is the point of effective stimulation for learners from the sensing rate values. We pay attention to the reaction frequency of sensor. The interval of sensor response is very active at the point around 20-30 seconds, but it gets less active after 30 seconds. This tells us that the learners make certain actions once every 30 seconds while they are studying. After 50 seconds, there is almost no reaction from the sensor We can see from Table 1 that the learner is moving actively when using SmartBox.

\subsection{Evaluation of SmartBox Functions}

In our experiments, we used SmartBox and measured the stimulation effects that the SmartBox has on learners. We checked the Smell Function, Light Function (high luminance LED) and Sound Function (different kind of music). In order to evaluate the effects of SmartBox on the learners,

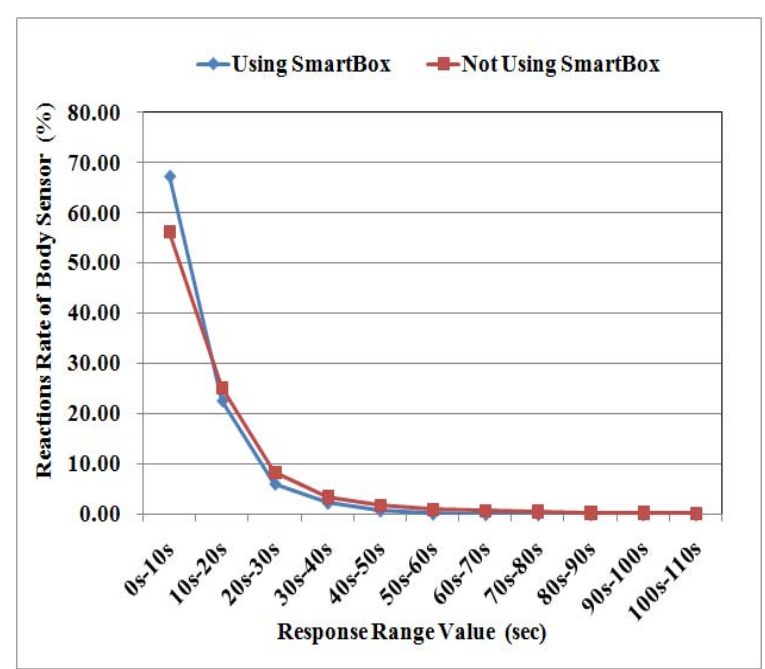

Figure 10. Reaction rate of body sensor.

we used human sensor of SmartBox and retrieved the information of the body movement. Using this information, we activated the following functions.

- Aroma Function

- Light Brightness Function

- Sound and Buzzer Function

The flowchart of learner's stimulation is shown in Fig. 11. In $[19,20,21]$, we found that the learners have a high concentration for about 20 minutes. After 20 minutes, when the system detects the body movement within 10 seconds, the system activates the Aroma Function. If the learners did not move for about 30 seconds, the system gives a slight stimulation by light. In the case when the learner did not move for about 40 seconds, the system gives the sound stimulation. The average response time for body sensor when using SmartBox and not using SmartBox is shown in Fig. 12. We can see that the reaction time when using SmartBox is better than not using SmartBox. We found that the sound stimulation has better effects in the learning process than the light stimulation. We gave the stimulation by light between 30 seconds and 40 seconds, but the learner's body was not moving. But, the learner's body moved more actively when we stimulated with the sound.

\subsection{Measurement of Learner's Body Movement During Study}

In order to check the effects of the SmartBox on the learner, high school students studying mathematics first used the SmartBox and in another experiment did not use the SmartBox.

We measured learners body movement as follows. 


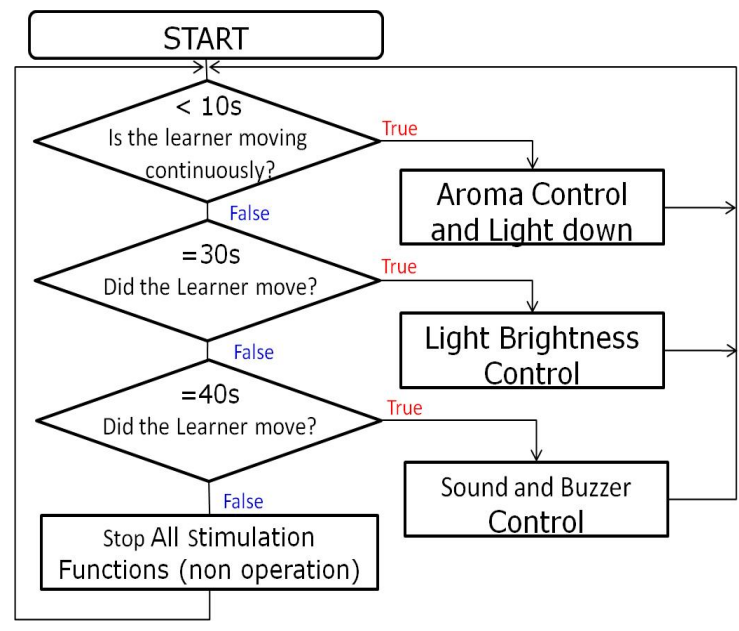

Figure 11. Flowchart of learner stimulation.

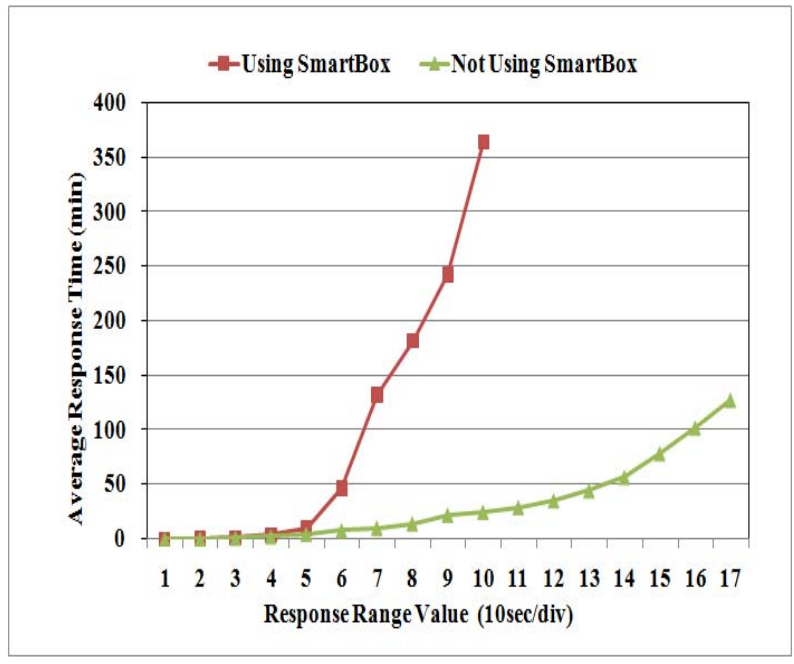

Figure 12. Average response time for body sensor when using SmartBox and not using SmartBox.

\section{- Using SmartBox}

Number of learners 24 people and the total measurement time about 24 hours.

\section{- Not Using SmartBox}

Number of learners 17 people and the total measurement time about 17 hours.

The average response time for learner's body movement for the cases when the learners used SmartBox and did not use the SmartBox are shown in Fig. 13. When the movement of learner's body is not detected for 30 seconds, we gave to

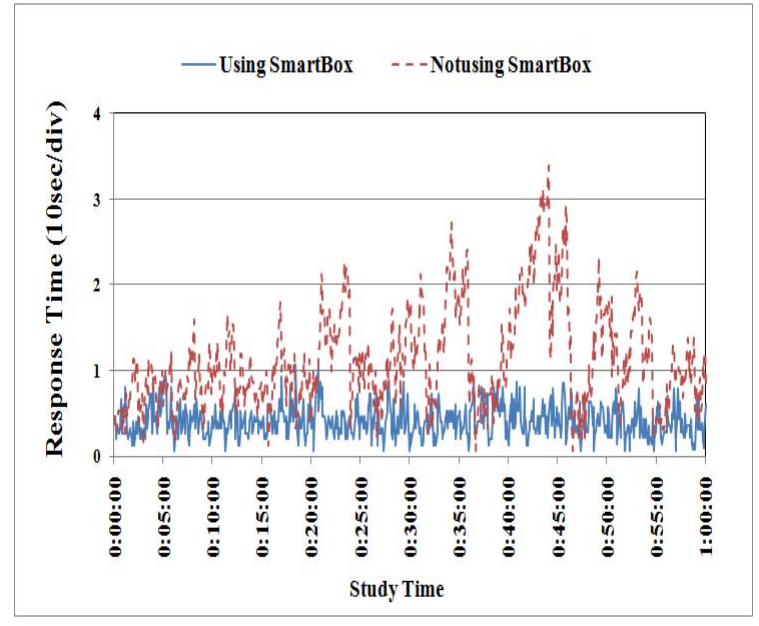

Figure 13. Average response time versus study time when using and not using SmartBox.

the learner a stimulation by light (High Brightness LED). At that time, the learner is regaining the concentration. Also, if the learner did not move for about 40 seconds, we gave the sound stimulation. The comparison between these two figures shows that the use of SmartBox is an effective way to improve the learner motivation, because the learners concentration is higher using the SmartBox.

\subsection{Amount of Study When Using and Not Using SmartBox}

For the investigation of the amount of study, 7 high school students used the system. We carried out the experiments for two cases: using SmartBox and not using SmartBox. Each experimental time was one hour. The experimental results are shown in Table 2. The values inside the table are the number of exercises per minute. The results show that when using the SmartBox the amount of study is higher comparing with the case of not using SmartBox. This is because by using the SmartBox the concentration of the learners is increased.

\section{Conclusions and Future Work}

In this work, we presented the experimental results and evaluation of SmartBox in e-learning P2P System. The proposed system is able to stimulate the learners in a WAN by using JXTA-Overlay system.

We carried out many experiments to evaluate the effects of SmartBox for e-learning. From the simulation results we concluded that the use of SmartBox is an effective way to increase the learner's concentration. We showed by experimental results the amount of study when using and not using 
Table 2. Amount of study.

\begin{tabular}{|c|c|c|}
\hline $\begin{array}{c}\text { Learners } \\
@\end{array}$ & $\begin{array}{c}\text { Amount of Study } \\
\text { Using SmartBox }\end{array}$ & $\begin{array}{c}\text { Amount of Study } \\
\text { Not Using SmartBox }\end{array}$ \\
\hline Learner 1 & 21.77 & 12.59 \\
\hline Learner 2 & 23.33 & 21.67 \\
\hline Learner 3 & 15.70 & 13.33 \\
\hline Learner 4 & 26.23 & 19.35 \\
\hline Learner 5 & 28.33 & 22.95 \\
\hline Learner 6 & 30.65 & 24.38 \\
\hline Learner 7 & 34.02 & 31.79 \\
\hline Average & 25.72 & 20.87 \\
\hline
\end{tabular}

SmartBox. We found that the learner solved more exercises when they used the SmartBox. This shows that the learners concentration was increasing using the SmartBox.

In the future, we plan to evaluate the proposed system for many learners. We also would like to evaluate the proposed system for different scenarios where monitoring and control of students in the wide area is important.

\section{References}

[1] F. Xhafa, R. Fernandez, T. Daradoumis, L. Barolli, S. Caballe, "Improvement of JXTA Protocols for Supporting Reliable Distributed Applications in P2P Systems", Proc. of NBiS-2007 (Regensburg, Germany), LNCS 4658, pp.345-354, September 2007.

[2] L. Barolli, F. Xhafa, A. Durresi, G. De Marco, "M3PS: A JXTA-based Multi-platform P2P System and Its Web Application Tools", International Journal of Web Information Systems, Vol. 2, No. 3/4, pp. 187-196, 2006.

[3] IBM Corporation, "Making P2P Interoperable: Creating JXTA Systems Design P2P Systems that Extend Beyond Traditional Network Boundaries", 2007.

[4] Sun Microsystems, "JXTA Java Standard Edition v2.5: Programmers Guide”, June 2007.

[5] H. Bal, H. Casanova, J. Dongarra, and S. Matsuoka, "Application-Level Tools", In Foster et al (Eds), The Grid: Blueprint for a New Computing Infrastructure, Chapter 24, pp. 463-489, Morgan Kaufmann, 2003.

[6] S. M. Lui and S.H. Kwok, "Interoperability of Peer-toPeer File Sharing Protocols", SIGecom Exch.Journal, Vol. 3, No. 3, pp. 25-33, ACM Press, New York, NY, USA, 2002.

[7] F. Le Fessant, S. Handurukande, A. M. Kermarrec, L. Massouli,E "Clustering in Peer-to-Peer File Sharing Workloads", Peer-to-Peer Systems III, Lecture Notes in Computer Science, Vol. 3279/2005, pp. 217-226, 2005.
[8] K. P. Gummadi, R. J. Dunn, S. Saroiu, S. D. Gribble, H. M. Levy, J. Zahorjan, "Measurement, Modeling, and Analysis of a Peer-to-Peer File-sharing Workload", Proc. of 19-th ACM Symposium on Operating Systems Principles, pp. 314-329, 2003.

[9] CALAT Project, Nippon Telegraph and Telephone Corporation, http://www.calat.com/.

[10] CALsurf, NTT Software Corporation, http://webbase. ntts.co.jp/.

[11] The University of The Air, http://www.u-air.ac.jp/.

[12] California Virtual University, http://www.california. edu/.

[13] WIDE University, School of Internet, http://www. sfc.wide.ad.jp/soi/contents.html.

[14] Blacboard, http://www.blackboard.com/webct.

[15] Open University of Catalonia, http://www.uoc. edu/portal/english/.

[16] L. Barolli, A. Koyama, "A Web-based Distance Learning System Using Cooperative Agents", Chapter in Encyclopedia of Online Learning and Technology, Idea Group Inc. Publishing, Vol. 4, pp.430-439, April 2005.

[17] L. Barolli, A. Koyama, A. Durresi, G. De Marco, "A Web-based E-learning System for Increasing Study Efficiency by Stimulating Learner Motivation", Journal of Information Systems Frontiers, Springer Publishers, Vol. 8, No. 4, pp. 297-306, September 2006.

[18] D. Brookshier, D. Govoni, N. Krishnan, J.C Soto, "JXTA: Java P2P Programming", Sams Publishing, 2002.

[19] K. Matsuo, L. Barolli, F. Xhafa, A. Koyama, A. Durresi, and M. Takizawa, "Implementation @ and Design of New Functions for a Web-Based E-learning System to Stimulate Learners Motivation", Proc. of CISIS2008 (Barcelona, Spain), pp. 513-518, March 2008.

[20] K. Matsuo, L. Barolli, F. Xhafa, A. Koyama, A. Durresi, M. Takizawa, "Design and Implementation of a JXTA-Overlay P2P System and Smart Box", Proc. of FINA-2008, AINA-2008 Workshops Proceedings (Okinawa, Japan), pp. 407-412, March 2008.

[21] K. Matsuo, L. Barolli, V. Kolici, F. Xhafa, A. Koyama, A. Durresi, "Stimulation Effects of SmartBox for Elearning Using JXTA-Overlay P2P System", Proc. of CISIS-2009 (Fukuoka, Japan), pp. 231-238, 2009. 